\title{
SEMIADJOINT FUNCTORS AND QUINTUPLES
}

\author{
ROBERT DAVIS
}

ABSTRACT. We obtain a generalization of adjoint functors and triples by axiomatizing the behavior of Albanese varieties, obtain a few basic properties, and show that abelian varieties form a generalized type of triplable category over complete abstract varieties.

1. Basic properties. Let $U: B \rightarrow a$ be a functor. We will say that $U$ has a left semiadjoint $V$ if there are an object function $V: \operatorname{Obj} Q$ $\rightarrow \mathrm{Obj} B$ and a functor $E: B \rightarrow A b$, where $A b$ is the category of abelian groups, such that $E B \subseteq$ Aut $(U B)$, and the following conditions are satisfied. For every object $A$ of $Q$, there is a fixed map $\alpha_{A}: A$ $\rightarrow U V A=F A$ such that $f: A \rightarrow U B$ implies the existence of unique maps $g: V A \rightarrow B$ and $c \in E B$ such that $f=c U(g) \alpha_{A}$. Furthermore, if $h: B_{1} \rightarrow B_{2}$ and $c \in E B_{1}$ then $E(h)(c) \circ U(h)=U(h) \circ c$. We will of ten write $h_{*}$ or even $U(h)_{*}$ for $E(h) . E B$ will be called the group of translations of $B$.

Note first that $V$ becomes a functor in the usual way. Let $f: A \rightarrow B$, $g: B \rightarrow C$ be maps of $a$; then $V(f)$ is the unique map in $B$ such that $\alpha_{B} f=c U V(f) \alpha_{A}$ for some $c \in E V B$. We will denote this $c$ by $c(f)$. Clearly $V\left(1_{A}\right)=1_{V A}$. Also $\alpha_{C} g=c(g) U V(g) \alpha_{B}$ and we have

$$
\begin{aligned}
\alpha_{C} g f & =c(g) U V(g) \alpha_{B} f \\
& =c(g) U V(g) c(f) U V(f) \alpha_{A} \\
& =c(g)(V(g)) *(c(f)) U(V(g) V(f)) \alpha_{\boldsymbol{A}}
\end{aligned}
$$

whence $V(g f)=V(g) V(f)$ and $V$ is a functor. Then $\alpha: 1_{a} \rightarrow U V=F$ is a seminatural transformation, that is, for each $f: A \rightarrow B$ there is a unique $c(f) \in E V B$ such that $\alpha_{B} f=c(f) F(f) \alpha_{A}$. We have also the result that $c(g f)=c(g) V(g) * c(f)$.

We obtain a back adjunction $\beta: V U \rightarrow 1_{B B}$ in the usual way; if $B \in \operatorname{Obj} B$ then $\beta_{B}: V U B \rightarrow B$ and $d_{B} \in E B$ are the unique maps such that $d_{B} U\left(\beta_{B}\right) \alpha_{U B}=1_{U B}$. Thus $U\left(\beta_{B}\right) \alpha_{U B}=d_{B}{ }^{-1} \in E B$. Now suppose that $f: A \rightarrow U B$; the unique maps $g: V A \rightarrow B$ and $c \in E B$ such that $f=c U(g) \alpha_{A}$ are given by the formulas $g=\beta_{B} V(f)$ and $c=d_{B}\left(\beta_{B}\right)_{*} c(f)$, since

Received by the editors June 12, 1970.

AMS 1970 subject classifications. Primary 18A40; Secondary $14 \mathrm{~K} 99$.

Key words and phrases. Adjoint functor, triple, abelian variety, semiadjoint, seminatural transformation. 


$$
\begin{aligned}
d_{B}\left(\beta_{B}\right)_{*} c(f) U\left(\beta_{B}\right) F(f) \alpha_{A} & =d_{B} U\left(\beta_{B}\right) c(f) F(f) \alpha_{A} \\
& =d_{B} U\left(\beta_{B}\right) \alpha_{U B} f \\
& =f .
\end{aligned}
$$

In contrast to $\alpha, \beta$ is a true natural transformation: if $g: B \rightarrow C$ in $Q$, $g \beta_{B}=\beta_{C} V U(g)$. To see this, we observe that

$$
U\left(g \beta_{B}\right) \alpha_{U B}=U(g) d_{B}^{-1}=g_{*}\left(d_{B}^{-1}\right) U(g)
$$

and

$$
\begin{aligned}
U\left(\beta_{C} V U(g)\right) \alpha_{U B} & =U\left(\beta_{C}\right) F U(g) \alpha_{U B} \\
& =U\left(\beta_{C}\right) c(U g)^{-1} \alpha_{U C} U(g) \\
& =\left(\beta_{C}\right)_{* c}(U g)^{-1} U\left(\beta_{C}\right) \alpha_{U C} U(g) \\
& =\left(\beta_{C}\right)_{* C}(U g)^{-1} d_{C}^{-1} U(g)
\end{aligned}
$$

from which we get

$$
U(g)=g_{*}\left(d_{B}\right) U\left(g \beta_{B}\right) \alpha_{U B}
$$

and

$$
U(g)=d_{C}\left(\beta_{C}\right)_{* C}(U g) U\left(\beta_{C} V U(g)\right) \alpha_{U B}
$$

whence $\beta_{C} V U(g)=g \beta_{B}$; also, $g_{*}\left(d_{B}\right)=d_{C} \circ\left(\beta_{C}\right)_{*} c(U g)$. Furthermore, $\beta$ acts almost like an ordinary back adjunction. If $g: V A \rightarrow B$, there is at least one map $j: A \rightarrow U B$ such that $\beta_{B} V(j)=g$, namely $j=U(g) \alpha_{A}$. To see this, note that $\beta_{B} V(j)=\beta_{B} V U(g) V\left(\alpha_{A}\right)=g \beta_{V A} V\left(\alpha_{A}\right)$, so we need only show that $\beta_{V A} V\left(\alpha_{A}\right)=1_{V A}$. But

$$
\begin{aligned}
U\left(\beta_{V A} V\left(\alpha_{A}\right)\right) \alpha_{A} & =U\left(\beta_{V A}\right) F\left(\alpha_{A}\right) \alpha_{A} \\
& =U\left(\beta_{V A}\right) c\left(\alpha_{A}\right)^{-1} \alpha_{F A} \alpha_{A} \\
& =\left(\beta_{V A}\right)_{*} c\left(\alpha_{A}\right)^{-1} U\left(\beta_{V A}\right) \alpha_{F A} \alpha_{A} \\
& =\left(\beta_{V A}\right)_{*} c\left(\alpha_{A}\right)^{-1} \circ d_{V A}^{-1} \alpha_{A}
\end{aligned}
$$

so $\alpha_{A}=\left(\beta_{V A}\right)_{*}\left(c\left(\alpha_{A}\right)\right) d_{V A} U\left(\beta_{V A} V\left(\alpha_{A}\right)\right) \alpha_{A}$ and this implies that $\beta_{V A} V\left(\alpha_{A}\right)=1_{V A}$ and also that $d_{V A}^{-1}=\left(\beta_{V A}\right)_{* c}\left(\alpha_{A}\right)$.

Further miscellaneous consequences of the definitions which we shall need are: first, if $c \in E V U B$ then

$$
\begin{aligned}
\left(\beta_{B}\right)_{*}(c) & =\left(\beta_{B}\right)_{*}(c) 1_{U B}=\left(\beta_{B}\right)_{*}(c) U\left(\beta_{B}\right) \alpha_{U B} d_{B} \\
& =\beta_{B} c \alpha_{U B} d_{B} .
\end{aligned}
$$

Next, let $c \in E B$; then 


$$
\begin{aligned}
\left(\beta_{B}\right)_{* c}(c) U\left(\beta_{B} V(c)\right) \alpha_{U B} & =U\left(\beta_{B}\right) c(c) F(c) \alpha_{U B} \\
& =U\left(\beta_{B}\right) \alpha_{U B} c \\
& =d_{B}^{-1} c=c d_{B}^{-1} \\
& =c U\left(\beta_{B}\right) \alpha_{U B}
\end{aligned}
$$

which implies that $\beta_{B}=\beta_{B} V(c)$ and also that $c=\left(\beta_{B}\right)_{*} c(c)$, i.e., $U\left(\beta_{B}\right) c(c)=c U\left(\beta_{B}\right)$. Finally, suppose that $d \in E B, f: A \rightarrow U B$, and $d f=f$. Then there exist unique $c \in E B$ and $g: V A \rightarrow B$ such that $c U(g) \alpha_{A}$ $=f$; then $d c U(g) \alpha_{A}=c U(g) \alpha_{A}$ implies $d c=c$, or $d=1_{U B}$. Thus a translation other than 1 has no "fixed points."

Now we are ready to define the analog for semiadjoints of the concept of triple for ordinary adjoints.

Definition. A quintuple $Q=(F, \alpha, \mu, G, c)$ on a category $a$ consists of a functor $F: a \rightarrow a$, a functor $G: a \rightarrow A b$ such that $G A \subseteq \operatorname{Aut}(F A)$, a natural transformation $\mu: F^{2} \rightarrow F$, and a seminatural transformation $\alpha: 1_{\alpha} \rightarrow F$ where, if $f: A \rightarrow B, \alpha_{B J}=c(f) F(f) \alpha_{A}$ for a unique $c(f)$ in $G B$; also, $\mu \circ F \alpha=1, \mu \circ F \mu=\mu \circ \mu F$, and $\mu_{A} \alpha_{F A} \in G A$. We write $d_{\bar{V} A}^{-1}$ for $\mu_{A} \alpha_{F A}$. In addition, we require that: if $d \in G F A$, then $\mu_{A} d=$ $\mu_{A} d \alpha_{F A} d_{V A} \mu_{A} ;$ if $c \in G A$ then $c \mu_{A}=\mu_{A} c(c)$ and $\mu_{A} F(c)=\mu_{A}$; and $f: B \rightarrow F A, c f=f$ imply $c=1_{F A}$. An algebra over $Q$ is a pair $(A, \xi)$ where $\xi: F A \rightarrow A, \xi F(\xi)=\xi \mu_{A}$, and $\xi \alpha_{A} \in \operatorname{Aut}(A)$. We write $d_{A}^{-1}$ for $\xi \alpha_{A}$ and $\xi_{*}(d)$ for $\xi d \alpha_{A} d_{A}$ for each $d \in G A$; and we require that there be for each $d$ a map $c: A \rightarrow A$ such that $\xi d=c \xi$; and that if $c \in \xi_{*} G A$ then $c \xi=\xi c(c)$ and $\xi F(c)=\xi$, and $c f=f$ implies $c=1_{A}$ for all maps $f$ with codomain $A$. A homomorphism $f:(A, \xi) \rightarrow(B, \zeta)$ is a map $f: A \rightarrow B$ such that $\zeta F(f)$ $=f \xi$. The category of $Q$-algebras and homomorphisms is denoted by $a^{2}$.

Clearly a quintuple arises from any semiadjoint situation if we put $F=U V, G=E V, \mu=U \beta V$. Conversely, we have:

Proposition 1. Define $U: a^{8} \rightarrow a$ by $U(A, \xi)=A, U(f)=f$, and $V: a \rightarrow a^{Q}$ by $V A=\left(F A, \mu_{A}\right), V(f)=F(f)$. Then $V$ is left semiadjoint to $U$.

Proof. We first begin to construct the functor $E$. Put $E(A, \xi)$ $=\xi_{*} G A$ and $E(\xi)=\xi_{*}$. Then $\xi_{*}(d) \xi=\xi d$, for $d \in G A$, since $\xi d \alpha_{A} d_{A} \xi$ $=c \xi \alpha_{A} d_{A} \xi=c \xi=\xi d$. Furthermore, $d_{A}=\xi_{*}\left(d_{V A} c(\xi)^{-1}\right)$, since if $d_{A}$ is defined by this equation, we have

$$
\begin{aligned}
\xi \alpha_{A} d_{A} \xi & =\xi \alpha_{A} \xi d_{V A} c(\xi)^{-1}=\xi c(\xi) F(\xi) \alpha_{F A} d_{V A} c(\xi)^{-1} \\
& =\xi_{*} c(\xi) \xi F(\xi) \alpha_{F A} d_{V A} c(\xi)^{-1}=\xi \xi \mu_{A} \alpha_{F A} d_{V A} c(\xi)^{-1} \\
& =\xi_{*} c(\xi) \xi c(\xi)^{-1}=\xi=1_{A} \xi
\end{aligned}
$$


so $\xi \alpha_{A} d_{A}=1_{A}$ since $\xi$ is epic. Thus, $d_{A} \in E(A, \xi)$ as required.

Now, let $(B, \zeta)$ be an algebra and $f: A \rightarrow B$. There exist maps $g: V A \rightarrow(B, \zeta)$ and $c \in E(B, \zeta)$ such that $f=c g \alpha_{A}$, namely $g=\zeta F(f)$ and $c=d_{B} \zeta_{*} c(f)$. We must show that $c$ and $g$ are unique; it is this step which requires the extra conditions imposed on $\zeta$ in the definition. Suppose $c g \alpha_{A}=d h \alpha_{A}$ where $h: V A \rightarrow(B, \zeta)$ and $d \in E(B, \zeta)$. By multiplying both sides by $d^{-1}$ we may write the equation in the form $\operatorname{cg} \alpha_{A}=h \alpha_{A} ;$ then

$$
\begin{aligned}
h & =h \mu_{A} F\left(\alpha_{A}\right)=\zeta F(h) F\left(\alpha_{A}\right)=\zeta F\left(c g \alpha_{A}\right) \\
& =\zeta F(c) F\left(g \alpha_{A}\right)=\zeta F(g) F\left(\alpha_{A}\right) \\
& =g \mu_{A} F\left(\alpha_{A}\right)=g,
\end{aligned}
$$

so $c g \alpha_{A}=g \alpha_{A}$ and $c=1_{B}$.

Next let $f:(A, \xi) \rightarrow(B, \zeta)$ be a homomorphism. Since $E$ is to be a functor, the map $f_{*}$ will have to satisfy $\zeta_{*} G(f)=\zeta_{*} F(f)_{*}=f_{*} \xi_{*}$, and since $\zeta_{*}$ is epic this equation will define $f_{*}$ provided it defines it consistently, i.e., provided $\zeta_{*}(c)=\zeta_{*}(d)$ implies $\zeta_{*} F(f)_{*}(c)=\zeta_{*} F(f)_{*}(d)$. But $\xi c \alpha_{A} d_{A}=\xi_{*}(c)=\xi_{*}(d)=\xi d \alpha_{A} d_{A}$ so $\xi c \alpha_{A}=\xi d \alpha_{A}$

$$
\begin{aligned}
f \xi c \alpha_{A} & =f \xi d \alpha_{A} \\
\zeta F(f) c \alpha_{A} & =\zeta F(f) d \alpha_{A} \\
\zeta_{*} F(f)_{*}(c) \zeta F(f) \alpha_{A} & \left.=\zeta_{*} F(f)\right)_{*}(d) \zeta F(f) \alpha_{A}
\end{aligned}
$$

and therefore $\zeta_{*} F(f)_{*}(c)=\zeta_{*} F(f)_{*}(d)$. Next, write $c=\xi_{*}(d)$; we must verify that $f c=f_{*}(c) f$. We have $f_{*}(c) f \xi=f_{*} \xi_{*}(d) f \xi=\zeta_{*} F(f)_{*}(d) \zeta F(f)$ $=\zeta F(f) d=f \xi d=f \xi_{*}(d) \xi=f c \xi$ and the desired equation follows since $\xi$ is epic. Now, $\xi_{*}$ is a homomorphism since $\xi_{*}(c d) \xi=\xi c d=\xi_{*}(c) \xi d=\xi_{*}(c)$ $\xi_{*}(d) \xi$ and $\xi$ is epic; since $\zeta_{*}, F(f)_{*}=G(f)$, and $\xi_{*}$ are all homomorphisms, so is $f_{*}$. It is straightforward that $(g f)_{*}=g_{*} f_{*}$. Thus $E: a^{8}$ $\rightarrow \mathrm{Ab}$ is a functor.

2. Products. The preservation properties, etc., of ordinary adjunctions do not carry over easily to the case of semiadjunctions. However, we can prove:

Proposition 2. Let $a$ be a category with finite products and $Q$ $=(F, \alpha, \mu, G, c)$ a quintuple on a with the property that $c_{A} \in E(A, \xi)$, $c_{B} \in E(B, \zeta), p_{A}: A \times B \rightarrow A$ implies $G\left(p_{A}\right)\left(c\left(c_{A} \times c_{B}\right)\right)=c\left(c_{A}\right)$. Then $a^{Q}$ has finite products which are preserved by $U$.

Proof. We define $(A, \xi) \times(B, \zeta)=(A \times B, \rho)$ where $\rho=(\xi \times \zeta)$ - $\left(F p_{A}, F p_{B}\right): F(A \times B) \rightarrow A \times B$. There are six conditions on $\rho$ to be verified. One sees straightforwardly that $\rho \alpha_{A \times B}=\left(\xi_{*}\left(c\left(p_{A}\right)\right) d_{A}^{-1}\right)$ 
$\times\left(\zeta_{*}\left(c\left(p_{B}\right)\right) d_{B}^{-1}\right) \in \operatorname{Aut}(A \times B)$. The condition $\rho F(\rho)=\rho \mu_{A \times B}$ follows as in the case of ordinary triples. If $d \in G(A \times B)$, it is easily seen that $\rho_{*}(d)=\left(\xi F p_{A}\right)_{*}(d) \times\left(\zeta F p_{B}\right)_{*}(d)$; thus, it follows that $E(A \times B, \rho)$ $=E(A, \xi) \times E(B, \zeta)$. Then $p_{A} \rho F\left(c_{A} \times c_{B}\right)=\xi F\left(c_{A}\right) F\left(p_{A}\right)=\xi F\left(p_{A}\right)=p_{A} \rho$ and similarly $p_{B} \rho F\left(c_{A} \times c_{B}\right)=p_{B} \rho$ so $\rho F\left(c_{A} \times c_{B}\right)=\rho$. Suppose $f$ $=(g, h): C \rightarrow A \times B$ and $\left(c_{A} \times c_{B}\right) f=f$. Then $(g, h)=\left(c_{A} \times c_{B}\right)(g, h)$ $=\left(c_{A} g, c_{B} h\right)$ so $c_{A}=1_{A}, c_{B}=1_{B}$. Only the remaining condition requires our hypothesis on $G\left(p_{A}\right)$, namely that $\left(c_{A} \times c_{B}\right) \rho=\rho c\left(c_{A} \times c_{B}\right)$. For this, we have $p_{A}\left(c_{A} \times c_{B}\right)=p_{A}(\xi \times \zeta)\left(c\left(c_{A}\right) \times c\left(c_{B}\right)\right)\left(F p_{A}, \quad F p_{B}\right)=$ $\xi c\left(c_{A}\right) F\left(p_{A}\right)=\xi F\left(p_{A}\right) c\left(c_{A} \times c_{B}\right)=p_{A} \rho c\left(c_{A} \times c_{B}\right)$ and similarly for $p_{B}$. Hence $(A \times B, \rho)$ is an object of $Q^{Q}$ and that it serves as the product follows as in the case of triples.

3. Abelian groups in categories. The standard example of a semiadjoint situation (see [1]) has $Q=$ category of abstract varieties and everywhere-defined rational maps, $B=$ category of Abelian varieties, $U B=$ the underlying variety of $B, E B=$ the underlying group of $B, V A=$ the Albanese variety of $A$. We will use for $\propto$ instead the category of complete varieties and approach axiomatically the question of whether $B \cong Q^{Q}$.

Thus, let $a$ be a category with finite products and a terminal object $T$, and let $B$ be the category of abelian group structures on the objects of $a$. Suppose $U: B \rightarrow Q$ has a left semiadjoint $V$ and let $Q$ be the induced quintuple. We will write $t_{A}: A \rightarrow T$ and $e_{B}: T \rightarrow B$ for the unique maps. The obvious functor $B \rightarrow Q^{\beta}$ is faithful; we will show it is full and representative. Let $(A, \xi)$ be any $\boldsymbol{Q}$-algebra.

LeMma. $\xi_{*}\left(\left(F d_{A}\right)_{*}(c(\xi)) d_{V A}^{-1} c\left(d_{A}\right)\right)=1_{A}$.

Proof. We know that $\xi_{*}\left(d_{V A}\right)=d_{A} \xi_{*}(c(\xi))$ so the equation is equivalent to

$$
\begin{aligned}
d_{A} & =\xi_{*}\left(\left(F d_{A}\right)_{*}(c(\xi)) c(\xi)^{-1} c\left(d_{A}\right)\right) \\
& =d_{A} \xi_{*}\left(F d_{A}\right)_{*} c(\xi) \xi_{*} c(\xi)^{-1}
\end{aligned}
$$

or $\xi_{*}(c(\xi))=\xi_{*}\left(F d_{A}\right)_{*} c(\xi)$. This is equivalent to $\xi c(\xi)=\xi F\left(d_{A}\right) c(\xi)$ which is true since $\xi=\xi F\left(d_{A}\right)$.

Proposition 3. $B$ is equivalent to $a^{8}$; in particular, the category of abelian varieties is "quintuplable" over the category of complete abstract varieties.

Proof. First we show that there is an abelian group structure on $A$ making $\xi: F A \rightarrow A$ a homomorphism. Clearly since $\xi$ is a retraction there is at most one such structure. Write $m_{F A}: F A \times F A \rightarrow F A$ for the 
multiplication, $i_{F A}: A \rightarrow A$ for the inverse and $e_{F A}: T \rightarrow F A$ for the identity. Define $m_{A}=\xi m_{F A}\left(\alpha_{A} d_{A} \times \alpha_{A} d_{A}\right), i_{A}=\xi i_{F A} \alpha_{A} d_{A}, e_{A}=\xi e_{F A}$. The verifications of the group laws on $A$, of the fact that $\xi$ is a homomorphism, and that any homomorphism $f:(A, \xi) \rightarrow(B, \zeta)$ is a homomorphism on the induced group structures are all variations of the same technique, so we will prove only the identity $m_{A}\left(i_{A}, 1_{A}\right)$ $=e_{A} t_{A}$. Recall that $m_{F A}$ is a homomorphism. Then:

$$
\begin{aligned}
m_{A}\left(i_{A}, 1_{A}\right)(\xi & =\xi m_{F A}\left(\alpha_{A} d_{A} \xi i_{F A} \alpha_{A} d_{A} \xi, \alpha_{A} d_{A} \xi\right) \\
& =\xi m_{F A}\left(F(\xi) k i_{F A} F(\xi) k, F(\xi) k\right) \\
& =\xi F(\xi) m_{F F A}(k, k)\left(i_{F A} F(\xi) k, 1_{F A}\right) \\
& =\xi \mu_{A} m_{F F A}(k, k)\left(i_{F A} F(\xi) k, 1_{F A}\right) \\
& =\xi m_{F A}\left(\mu_{A} k, \mu_{A} k\right)\left(i_{F A} F(\xi) k, 1_{F A}\right) \\
& =\xi m_{F A}(c, c)\left(i_{F A} F(\xi) k, 1_{F A}\right) \\
& =\xi *\left(c^{2}\right) \xi m_{F A}\left(i_{F A}, 1_{F A}\right)\left(F(\xi) k, 1_{F A}\right) \\
& =1_{A}^{2} \xi e_{F A} t_{F A}\left(F(\xi) k, 1_{F A}\right) \\
& =\xi e_{F A} t_{F A}=e_{A} t_{F A}=e_{A} t_{A}(\xi)
\end{aligned}
$$

where $k=\left(F\left(\alpha_{A} d_{A}\right)\right)_{*} c(\xi) \alpha_{F A} c\left(d_{A}\right)$ and $c=\left(F d_{A}\right)_{*}(c(\xi)) d_{V A}^{-1} c\left(d_{A}\right)$, and we have applied the lemma. Now $\xi$ is epic since it is a retraction, so we get $m_{A}\left(i_{A}, 1_{A}\right)=e_{A} t_{A}$ as required.

\section{REFERENCE}

1. Serge Lang, Abelian varieties, Interscience Tracts in Pure and Appl. Math., no. 7, Interscience, New York, 1959. MR 21 \#4959.

Southern Methodist University, Dallas, Texas 75222 